\title{
Temperature Dependent Resistivity of Chiral Single-Walled Carbon Nanotubes in the Presence of Coherent Light Source
}

\author{
Anthony Twum ${ }^{1 *}$ (), Raymond Edziah ${ }^{1}$, Samuel Yeboah Mensah', Kwadwo Dompreh ${ }^{1}$, \\ Patrick Mensah-Amoah', Augustine Arthur'1, Natalia G. Mensah², Kofi Adu ${ }^{3}$, \\ George Nkrumah-Buandoh ${ }^{4}$ \\ ${ }^{1}$ Department of Physics, University of Cape Coast, Cape Coast, Ghana \\ ${ }^{2}$ Department of Mathematics, University of Cape Coast, Cape Coast, Ghana \\ ${ }^{3}$ Department of Physics, The Pennsylvania State University-Altoona College, Altoona, USA \\ ${ }^{4}$ Department of Physics, University of Ghana, Accra, Ghana \\ Email: *atwum@ucc.edu.gh
}

How to cite this paper: Twum, A., Edziah, R., Mensah, S.Y., Dompreh, K., Mensah-Amoah, P., Arthur, A., Mensah, N.G., Adu, K. and Nkrumah-Buandoh, G. (2021) Temperature Dependent Resistivity of Chiral Single-Walled Carbon Nanotubes in the Presence of Coherent Light Source. World Journal of Condensed Matter Physics, $11,77-86$

https://doi.org/10.4236/wjcmp.2021.114006

Received: October 18, 2021

Accepted: November 27, 2021

Published: November 30, 2021

Copyright $\odot 2021$ by author(s) and Scientific Research Publishing Inc. This work is licensed under the Creative Commons Attribution International License (CC BY 4.0).

http://creativecommons.org/licenses/by/4.0/

\begin{abstract}
We have studied the axial resistivity of chiral single-walled carbon nanotubes (SWCNTs) in the presence of a combined direct current and high frequency alternating fields. We employed semiclassical Boltzmann equations approach and compared our results with a similar study that examined the circumferential resistivity of these unique materials. Our work shows that these materials display similar resistivity for both axial and circumferential directions and this largely depends on temperature, intensities of the applied fields and material parameters such as chiral angle. Based on these low-temperature bidirectional conductivity responses, we propose chiral SWCNTs for design of efficient optoelectronic devices.
\end{abstract}

\section{Keywords}

Chiral Single-Wall Carbon Nanotubes, Boltzmann Transport Equation, Axial Resistivity, Chiral Angle

\section{Introduction}

Since the accidental discovery of carbon nanotubes (CNTs) and their fabrication by Iijima in the early 1990s [1], there has been a lot of research interest in this novel material. Several research works have reported practical usefulness of CNTs as composites, catalysts and molecular wires. Essentially, CNTs are tiny and single sheets of carbon atom rolled into seamlessly meshed tubes at the nanoscale 
level [2]. The unique material properties of CNTs are their characteristic electrical and thermal conductivities. A CNT is either single-walled or multi-walled. Single-walled carbon nanotubes (SWCNTs) consist of a roll of one graphene sheet. The electrical behaviour of SWCNTs is determined by their chirality [3]. Researchers in [4] studied the electrical behaviour of multi-walled carbon nanotubes (MWCNTs) network embedded in amorphous silicon nitride. As part of their findings, they reported that the resistance-temperature characteristic is practically linear except at very low temperatures. This suggests a metallic behaviour of the MWCNT networks. Experimental investigations have also been carried out on the scaling of resistance and electron mean-free-path of SWCNTs [5]. Furthermore, the authors in [5] determined the length-dependent resistance of SWCNTs and also considered the variation of resistivity with temperature. The temperature dependent resistivity of armchair CNTs has also been studied experimentally [6] and the results clearly indicated metallic behavior of the SWCNT at high temperatures with resistivity increasing approximately linearly with temperature.

In this paper, we studied the temperature dependent resistivity of SWCNTs along their tubular axes in the presence of a combined direct current and laser radiation. Our results are compared with similar findings reported in reference [7] which reported on resistivity of these novel materials along the base helix.

\section{Theory}

Using semi-classical Boltzmann transport equation (BTE) with constant electron relaxation time, the carrier current density and axial resistivity in the SWCNT is evaluated as functions of the geometric chiral angle $\theta_{h}$, temperature $T$, the real overlapping integrals for jumps along the nanotube axis $\Delta_{z}$ and the base helix $\Delta_{s}$. This is done by following the approach of [7] [8] together with the phenomenological model of a SWCNT developed in references [9] [10]. Employing BTE, a chiral SWCNT placed in an electric field applied along the nanotube axisis studied under a temperature gradient $\nabla T$ such that

$$
\frac{\partial f(r, p, t)}{\partial t}+v(p) \frac{\partial f(r, p, t)}{\partial r}+e E(t) \frac{\partial f(r, p, t)}{\partial p}=-\frac{f(r, p, t)-f_{0}(p)}{\tau}
$$

where $f(r, p, t)$ is the distribution function, $f_{0}(p)$ is the equilibrium distribution function, $v(p)$ is the electron velocity, $r$ is the electron position, $p$ is the electron dynamical momentum, $t$ is time elapsed, $\tau$ is the electron relaxation time which is assumed to be constant and $e$ is the electron charge. The applied dc-ac field, $E(t)=E_{0}+E_{1} \cos (w t)$, where $E_{0}$ is the constant electric field, $E_{1}$ and $w$ are the amplitude and frequency of the ac field, respectively.

We employ the perturbation approach to solve Equation (1) by treating the second term on the left-hand side as a weak perturbation. In the linear approximation of $\nabla T$ and $\nabla \mu$, the solution to Equation (1) is

$$
f(p)=\tau^{-1} \int_{0}^{\infty} \exp \left(-\frac{t}{\tau}\right) f_{0}\left(p-e \int_{t-t^{\prime}}^{t}\left[E_{0}+E_{1} \cos w t^{\prime}\right] \mathrm{d} t^{\prime}\right) \mathrm{d} t
$$




$$
\begin{aligned}
& +\int_{0}^{\infty} \exp \left(-\frac{t}{\tau}\right) \mathrm{d} t\left\{\left[\varepsilon\left(p-e \int_{t-t^{\prime}}^{t}\left[E_{0}+E_{1} \cos w t^{\prime}\right] \mathrm{d} t^{\prime}\right)-\mu\right] \frac{\nabla T}{T}+\nabla \mu\right\} \\
& \times v\left(p-e \int_{t-t^{\prime}}^{t}\left[E_{0}+E_{1} \cos w t^{\prime}\right] \mathrm{d} t^{\prime}\right) \frac{\partial f_{0}}{\partial \varepsilon}\left(p-e \int_{t-t^{\prime}}^{t}\left[E_{0}+E_{1} \cos w t^{\prime}\right] \mathrm{d} t^{\prime}\right)
\end{aligned}
$$

where $\varepsilon(p)$ is the tight-binding energy of the electron and $\mu$ is the chemical potential.

The current density is expressed as

$$
j=e \sum_{p} v(p) f(p)
$$

Substituting Equation (2) into Equation (3) gives

$$
\begin{aligned}
j= & e \tau^{-1} \int_{0}^{\infty} \exp \left(-\frac{t}{\tau}\right) \mathrm{d} t \sum_{p} v(p) f_{0}\left(p-e \int_{t-t^{\prime}}^{t}\left[E_{0}+E_{1} \cos w t^{\prime}\right] \mathrm{d} t^{\prime}\right) \\
& +e \int_{0}^{\infty} \exp \left(-\frac{t}{\tau}\right) \mathrm{d} t \sum_{p} v(p)\left\{\left[\varepsilon\left(p-e \int_{t-t^{\prime}}^{t}\left[E_{0}+E_{1} \cos w t^{\prime}\right] \mathrm{d} t^{\prime}\right)-\mu\right] \frac{\nabla T}{T}+\nabla \mu\right\} \\
& \times v\left(p-e \int_{t-t^{\prime}}^{t}\left[E_{0}+E_{1} \cos w t^{\prime}\right] \mathrm{d} t^{\prime}\right) \frac{\partial f_{0}}{\partial \varepsilon}\left(p-e \int_{t-t^{\prime}}^{t}\left[E_{0}+E_{1} \cos w t^{\prime}\right] \mathrm{d} t^{\prime}\right)
\end{aligned}
$$

Employing the transformation

$$
p-e \int_{t-t^{\prime}}^{t}\left[E_{0}+E_{1} \cos w t^{\prime}\right] \mathrm{d} t^{\prime} \rightarrow p,
$$

Equation (4) becomes

$$
\begin{aligned}
j= & e \tau^{-1} \int_{0}^{\infty} \exp \left(-\frac{t}{\tau}\right) \mathrm{d} t \sum_{p} v\left(p-e \int_{t-t^{\prime}}^{t}\left[E_{0}+E_{1} \cos w t^{\prime}\right] \mathrm{d} t^{\prime}\right) f_{0}(p) \\
& +e \int_{0}^{\infty} \exp \left(-\frac{t}{\tau}\right) \mathrm{d} t \sum_{p}\left\{[\varepsilon(p)-\mu] \frac{\nabla T}{T}+\nabla \mu\right\} \\
& \times\left\{v(p) \frac{\partial f_{0}(p)}{\partial \varepsilon}\right\} v\left(p-e \int_{t-t^{\prime}}^{t}\left[E_{0}+E_{1} \cos w t^{\prime}\right] \mathrm{d} t^{\prime}\right)
\end{aligned}
$$

We employ the phenomenological approach of references [9] [10] by considering a SWCNT as an infinitely long periodic chain of carbon atoms wrapped along a base helix. The axial current density is decomposed into the form

$$
j_{z}=Z^{\prime}+Y^{\prime} \sin \theta_{h}
$$

where $Z$ and $Y$ are components of the current density along the nanotube axis and the base helix, respectively.

Furthermore, we neglect the interference between the axial and helical paths connecting a pair of atoms, quantization of transverse motion can be ignored [9] [10]; an approximation that best describes doped chiral carbon nanotubes [11].

Resolving the current density along the tubular axis (z-axis) and base helix, we obtain

$$
\begin{aligned}
Z^{\prime}= & e \tau^{-1} \int_{0}^{\infty} \exp \left(-\frac{t}{\tau}\right) \mathrm{d} t \sum_{p} v_{z}\left(p-e \int_{t-t^{\prime}}^{t}\left[E_{0}+E_{1} \cos w t^{\prime}\right] \mathrm{d} t^{\prime}\right) f_{0}(p) \\
& +e \int_{0}^{\infty} \exp \left(-\frac{t}{\tau}\right) \mathrm{d} t \sum_{p}\left\{[\varepsilon(p)-\mu] \frac{\nabla_{z} T}{T}+\nabla_{z} \mu\right\}
\end{aligned}
$$




$$
\times\left\{v_{z}(p) \frac{\partial f_{0}(p)}{\partial \varepsilon}\right\} v_{z}\left(p-e \int_{t-t^{\prime}}^{t}\left[E_{0}+E_{1} \cos w t^{\prime}\right] \mathrm{d} t^{\prime}\right)
$$

and

$$
\begin{aligned}
Y^{\prime}= & e \tau^{-1} \int_{0}^{\infty} \exp \left(-\frac{t}{\tau}\right) d t \sum_{p} v_{y}\left(p-e \int_{t-t^{\prime}}^{t}\left[E_{0}+E_{1} \cos w t^{\prime}\right] \mathrm{d} t^{\prime}\right) f_{0}(p) \\
& +e \int_{0}^{\infty} \exp \left(-\frac{t}{\tau}\right) \mathrm{d} t \sum_{p}\left\{[\varepsilon(p)-\mu] \frac{\nabla_{y} T}{T}+\nabla_{y} \mu\right\} \\
& \times\left\{v_{y}(p) \frac{\partial f_{0}(p)}{\partial \varepsilon}\right\} v_{y}\left(p-e \int_{t-t^{\prime}}^{t}\left[E_{0}+E_{1} \cos w t^{\prime}\right] \mathrm{d} t^{\prime}\right)
\end{aligned}
$$

Equations (7) and (8) are transformed using

$$
\sum_{p} \rightarrow \frac{2}{(2 \pi \hbar)^{2}} \int_{-\frac{\pi}{d_{y}}}^{\frac{\pi}{d_{y}}} \mathrm{~d} p_{y} \int_{-\frac{\pi}{d_{z}}}^{\frac{\pi}{d_{z}}} \mathrm{~d} p_{z}
$$

where $d_{z}$ and $d_{y}$ are the inter-atomic distance along the nanotube axis and the base helix respectively. Therefore, $Z$ 'and $Y^{\prime}$ become,

$$
\begin{aligned}
Z^{\prime}= & \frac{2 e \tau^{-1}}{(2 \pi \hbar)^{2}} \int_{0}^{\infty} \exp \left(-\frac{t}{\tau}\right) \mathrm{d} t \int_{-\frac{\pi}{d_{y}}}^{\frac{\pi}{d_{y}}} \mathrm{~d} p_{y} \int_{-\frac{\pi}{d_{z}}}^{\frac{\pi}{d_{z}}} \mathrm{~d} p_{z} v_{z}\left(p-e \int_{t-t^{\prime}}^{t}\left[E_{0}+E_{1} \cos w t^{\prime}\right] \mathrm{d} t^{\prime}\right) f_{0}(p) \\
& +\frac{2 e}{(2 \pi \hbar)^{2}} \int_{0}^{\infty} \exp \left(-\frac{t}{\tau}\right) \mathrm{d} t \int_{-\frac{\pi}{d_{y}}}^{\frac{\pi}{d_{y}}} \mathrm{~d} p_{y} \int_{-\frac{\pi}{d_{z}}}^{\frac{\pi}{d_{z}}} \mathrm{~d} p_{z}\left\{[\varepsilon(p)-\mu] \frac{\nabla_{z} T}{T}+\nabla_{z} \mu\right\} \\
& \times\left\{v_{z}(p) \frac{\partial f_{0}(p)}{\partial \varepsilon}\right\} v_{z}\left(p-e \int_{t-t^{\prime}}^{t}\left[E_{0}+E_{1} \cos w t^{\prime}\right] \mathrm{d} t^{\prime}\right)
\end{aligned}
$$

and

$$
\begin{aligned}
Y^{\prime}= & \frac{2 e \tau^{-1}}{(2 \pi \hbar)^{2}} \int_{0}^{\infty} \exp \left(-\frac{t}{\tau}\right) \mathrm{d} t \int_{-\frac{\pi}{d_{y}}}^{\frac{\pi}{d_{y}}} \mathrm{~d} p_{y} \int_{-\frac{\pi}{d_{z}}}^{\frac{\pi}{d_{z}}} \mathrm{~d} p_{z} v_{y}\left(p-e \int_{t-t^{\prime}}^{t}\left[E_{0}+E_{1} \cos w t^{\prime}\right] \mathrm{d} t^{\prime}\right) f_{0}(p) \\
& +\frac{2 e}{(2 \pi \hbar)^{2}} \int_{0}^{\infty} \exp \left(-\frac{t}{\tau}\right) \mathrm{d} t \int_{-\frac{\pi}{d_{y}}}^{\frac{\pi}{d_{y}}} \mathrm{~d} p_{y} \int_{-\frac{\pi}{d_{z}}}^{\frac{\pi}{d_{z}}} \mathrm{~d} p_{z}\left\{[\varepsilon(p)-\mu] \frac{\nabla_{y} T}{T}+\nabla_{y} \mu\right\} \\
& \times\left\{v_{y}(p) \frac{\partial f_{0}(p)}{\partial \varepsilon}\right\} v_{y}\left(p-e \int_{t-t^{\prime}}^{t}\left[E_{0}+E_{1} \cos w t^{\prime}\right] \mathrm{d} t^{\prime}\right)
\end{aligned}
$$

The integrals in Equations (9) and (10) were evaluated over the first Brillouin zone. The parameters $v, p, E, \nabla T$ and $\nabla \mu$ with subscripts $z$ and $y$ represent the respective components along the nanotube axis and along the base helix.

The energy dispersion relation for a chiral nanotube obtained in the tight-binding approximation is expressed as:

$$
\varepsilon(p)=\varepsilon_{0}-\Delta_{y} \cos \frac{p_{y} d_{y}}{\hbar}-\Delta_{z} \cos \frac{p_{z} d_{z}}{\hbar}
$$


where $\varepsilon_{0}$ is the energy of an outer-shell electron in an isolated carbon atom, $\Delta_{z}$ and $\Delta_{y}$ are the real overlapping integrals for jumps along the respective coordinates, $p_{y}$ and $p_{z}$ are the components of momentum tangential to the base helix and along the nanotube axis, respectively. The components $v_{y}$ and $v_{z}$ of the electron velocity $v$ are respectively.

$$
\begin{aligned}
& v_{y}(p)=\frac{\partial \varepsilon(p)}{\partial P_{y}}=\frac{\Delta_{y} d_{y}}{\hbar} \sin \frac{p_{y} d_{y}}{\hbar} \\
& v_{z}(p)=\frac{\partial \varepsilon(p)}{\partial P_{z}}=\frac{\Delta_{z} d_{z}}{\hbar} \sin \frac{p_{z} d_{z}}{\hbar}
\end{aligned}
$$

Also,

$$
\begin{aligned}
& v_{y}\left(p-e \int_{t-t^{\prime}}^{t}\left[E_{0}+E_{1} \cos w t^{\prime}\right] \mathrm{d} t^{\prime}\right) \\
& =\frac{\partial \varepsilon}{\partial P_{y}}\left(p-e \int_{t-t^{\prime}}^{t}\left[E_{0}+E_{1} \cos w t^{\prime}\right] \mathrm{d} t^{\prime}\right) \\
& =\frac{\Delta_{y} d_{y}}{\hbar}\left\{\sin \frac{p_{y} d_{y}}{\hbar} \cos \left(p-e \int_{t-t^{\prime}}^{t}\left[E_{0}+E_{1} \cos w t^{\prime}\right] \mathrm{d} t^{\prime}\right)\right. \\
& -\cos \frac{p_{y} d_{y}}{\hbar} \sin \left(p-e \int_{t-t^{\prime}}^{t}\left[E_{0}+E_{1} \cos w t^{\prime}\right] \mathrm{d} t^{\prime}\right)
\end{aligned}
$$

Similarly,

$$
\begin{aligned}
v_{z}= & \frac{\Delta_{z} d_{z}}{\hbar}\left\{\sin \frac{p_{z} d_{z}}{\hbar} \cos \left(p-e \int_{t-t^{\prime}}^{t}\left[E_{0}+E_{1} \cos w t^{\prime}\right] \mathrm{d} t^{\prime}\right)\right. \\
& -\cos \frac{p_{z} d_{z}}{\hbar} \sin \left(p-e \int_{t-t^{\prime}}^{t}\left[E_{0}+E_{1} \cos w t^{\prime}\right] \mathrm{d} t^{\prime}\right)
\end{aligned}
$$

The carrier density of the non-degenerate electron gas can be determined by expressing the Boltzmann equilibrium distribution function $f_{0}(p)$ as

$$
f_{0}(p)=C \exp \left(\frac{\Delta_{y} \cos \frac{p_{y} d_{y}}{\hbar}+\Delta_{z} \cos \frac{p_{z} d_{z}}{\hbar}+\mu-\varepsilon_{0}}{k T}\right)
$$

The normalization constant $C$ is expressed as

$$
C=\frac{d_{y} d_{z} n_{0}}{2 I_{0}\left(\Delta_{y}^{*}\right) I_{0}\left(\Delta_{z}^{*}\right)} \exp \left(-\frac{\mu-\varepsilon_{0}}{k T}\right)
$$

where $n_{0}$ is the surface charge density, $I_{n}(x)$ is the modified Bessel function of $n^{\text {th }}$ order,

$$
\Delta_{y}^{*}=\frac{\Delta_{y}}{k T}, \Delta_{z}^{*}=\frac{\Delta_{z}}{k T}
$$

and $k$ is Boltzmann's constant.

We substitute Equations (11)-(16) into Equations (9) and (10) and simplify the integrals to obtain

$$
Y^{\prime}=-\sigma_{y}(E) E_{y n}^{*}-\sigma_{y}(E) \frac{k}{e}\left\{\left(\frac{\varepsilon_{0}-\mu}{k T}\right)-\Delta_{y}^{*} \frac{I_{0}\left(\Delta_{y}^{*}\right)}{I_{1}\left(\Delta_{y}^{*}\right)}+2-\Delta_{z}^{*} \frac{I_{1}\left(\Delta_{z}^{*}\right)}{I_{0}\left(\Delta_{z}^{*}\right)}\right\} \nabla_{y} T
$$




$$
Z^{\prime}=-\sigma_{z}(E) E_{z n}^{*}-\sigma_{z}(E) \frac{k}{e}\left\{\left(\frac{\varepsilon_{0}-\mu}{k T}\right)-\Delta_{z}^{*} \frac{I_{0}\left(\Delta_{z}^{*}\right)}{I_{1}\left(\Delta_{z}^{*}\right)}+2-\Delta_{y}^{*} \frac{I_{1}\left(\Delta_{y}^{*}\right)}{I_{0}\left(\Delta_{y}^{*}\right)}\right\} \nabla_{z} T
$$

where we have defined $E_{y n}^{*}$ as

$$
E_{y n}^{*}=E_{n}+\nabla_{y} \frac{\mu}{e}, E_{s n}^{*}=E_{z n}^{*} \sin \theta_{\hbar}
$$

Also, the electrical conductivity $\sigma_{i}(E)$ is expressed as

$$
\sigma_{i}(E)=\frac{e^{2} \tau \Delta_{i} d_{i}^{2} n_{0}}{\hbar^{2}} \frac{I_{1}\left(\Delta_{i}^{*}\right)}{I_{0}\left(\Delta_{i}^{*}\right)} \sum_{n=-\infty}^{\infty} J_{n}^{2}(a)\left[\frac{1}{1+\left(\frac{e d_{i} E_{0}}{\hbar}+n w\right)^{2} \tau^{2}}\right], i=y, z
$$

Substituting Equations (17) and (18) into Equation (6), we obtain the following expression for axial the current density as

$$
\begin{aligned}
j_{z}= & -\left\{\sigma_{z}(E)+\sigma_{y}(E) \sin ^{2} \theta_{h}\right\} E_{z n}^{*} \\
& -\left\{\sigma_{z}(E) \frac{k}{e}\left[\left(\frac{\varepsilon_{0}-\mu}{k T}\right)-\Delta_{z}^{*} \frac{I_{0}\left(\Delta_{z}^{*}\right)}{I_{1}\left(\Delta_{z}^{*}\right)}+2-\Delta_{y}^{*} \frac{I_{1}\left(\Delta_{y}^{*}\right)}{I_{0}\left(\Delta_{y}^{*}\right)}\right]\right. \\
& \left.+\sigma_{y}(E) \frac{k}{e} \sin ^{2} \theta_{h}\left[\left(\frac{\varepsilon_{0}-\mu}{k T}\right)-\Delta_{y}^{*} \frac{I_{0}\left(\Delta_{y}^{*}\right)}{I_{1}\left(\Delta_{y}^{*}\right)}+2-\Delta_{z}^{*} \frac{I_{1}\left(\Delta_{z}^{*}\right)}{I_{0}\left(\Delta_{z}^{*}\right)}\right]\right\} \nabla_{z} T
\end{aligned}
$$

Employing the following definitions

$$
\xi=\frac{\varepsilon_{0}-\mu}{k T}, \quad A_{i}=\frac{I_{1}\left(\Delta_{i}^{*}\right)}{I_{0}\left(\Delta_{i}^{*}\right)}, \quad B_{i}=\frac{I_{0}\left(\Delta_{i}^{*}\right)}{I_{1}\left(\Delta_{i}^{*}\right)}-\frac{2}{\Delta_{i}^{*}}, \quad i=y, z
$$

Equation (20) becomes

$$
\begin{aligned}
j_{z}= & -\left\{\sigma_{z}(E)+\sigma_{y}(E) \sin ^{2} \theta_{h}\right\} E_{z n}^{*}-\left\{\sigma_{z}(E) \frac{k}{e}\left[\xi-\Delta_{z}^{*} B_{z}-\Delta_{y}^{*} A_{y}\right]\right. \\
& \left.+\sigma_{y}(E) \frac{k}{e} \sin ^{2} \theta_{h}\left[\xi-\Delta_{y}^{*} B_{y}-\Delta_{z}^{*} A_{z}\right]\right\} \nabla_{z} T
\end{aligned}
$$

Equation (21) defines the current density and the axial component of the electrical conductivity is the coefficient of the electric field $E_{z n}^{*}$ given as

$$
\sigma_{z z}=\sigma_{z}(E)+\sigma_{y}(E) \sin ^{2} \theta_{h}
$$

The resistivity of the chiral SWCNT along its axial direction is defined as

$$
\rho_{z}=\frac{1}{\sigma_{z}(E)+\sigma_{y}(E) \sin ^{2} \theta_{h}}
$$

\section{Results and Discussion}

In this work, we employed BTE to analytically study the axial electrical resistivity of a chiral SWCNT and obtained an expression for the axial component of the electrical resistivity, $\rho_{z}$ in the presence of applied field $E$ as shown in Equation (23). The $\rho_{z}$ expression was analyzed numerically by considering a chiral SWCNT 
having the following parameters: $d_{y}=1 \AA, d_{z}=2 \AA, \tau=0.3 \times 10^{-12} \mathrm{~s}$ and $\theta_{h}=4^{\circ}$, and $E_{0}=6.9063 \times 10^{7} \mathrm{~V} / \mathrm{m}, W=10^{12} \mathrm{~s}^{-1}$ and $E_{1}=5 \times 10^{7} \mathrm{~V} / \mathrm{m}$.

Figure 1(a) shows the dependence of the axial electrical resistivity, $\rho_{z}$ on temperature $T$, for various fixed values of the d.c. field, $E_{0}$. It is observed that at low temperatures up to about $100 \mathrm{~K}, \rho_{z}$ changes slowly with temperature and the temperature dependence of $\rho_{z}$ becomes generally linear with increasing temperature. This observation is attributed to electron-phonon interactions which cause scattering of the charge carriers along the tubular axis of the chiral SWCNT as temperature increases. The relatively low values of resistivity of SWCNT observed in Figure 1 clearly suggest that chiral SWCNTs do exhibit metallic properties. As observed in the case of circumferential resistivity [7], our results similarly reveal that as the electric field strength, $E_{0}$ increases the axial resistivity increases significantly. This temperature dependence resistivity response of SWCNTs has been observed experimentally [6] [12] [13]. This behaviour can be explained based on the fact that as $E_{0}$ is increased, the carbon atoms forming the walls of the SWCNT become more energized and tend to vibrate faster at larger amplitudes resulting in enhanced scattering of the electrons. Our study has also revealed that the resistivity of the SWCNTs decreases markedly with increasing $\Delta_{y}$ as shown in Figure 1(b).

Figure 2 shows the variation of the axial current density with temperature for selected values of the chiral angle, $\theta_{h}$ and dimensionless parameter $\Delta_{z}$. Increasing $\theta_{h}$ results in a decrease of the resistivity of the chiral SWCNT as seen in Figure 2(a), a behaviour similar to that observed for Figure 2(a) reveals that increasing the chiral angle, $\theta_{h}$ results in a decrease of the resistivity of the chiral SWCNT. On the other hand, Figure 2(b) shows that keeping $\Delta_{y}$ constant and varying $\Delta_{z}$ decreases $\rho_{z}$ by an order of magnitude. Also observed in Figure 2(b) is that $\rho_{z}$ remains unchanged for all values of $\Delta_{z}$ at temperatures below $100 \mathrm{~K}$ but decreases steadily by small margins with increasing temperature.
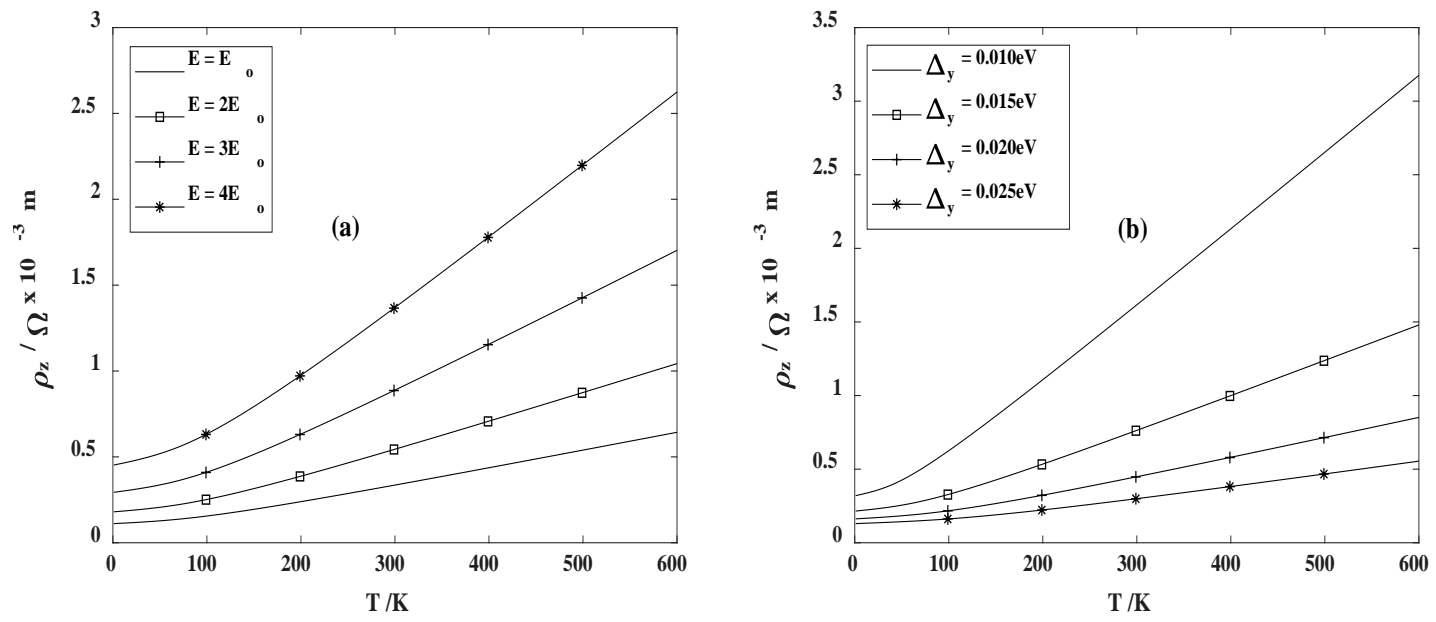

Figure 1. Dependence of axial current density $\left(\rho_{z}\right)$ on temperature $(T)$ for: (a) fixed values of the d.c. field $E_{0}$, $2 E_{0}, 3 E_{0}$ and $4 E_{0}$, where $E_{0}=6.9063 \times 10^{7} \mathrm{~V} / \mathrm{m} . E_{1}=5.0 \times 10^{7} \mathrm{~V} / \mathrm{m}, \Delta_{y}=0.018 \mathrm{eV}$ and $\Delta_{z}=0.024 \mathrm{eV}$. (b) Fixed values of $\Delta_{y} . \Delta_{z}=0.024 \mathrm{eV}, E_{1}=5.0 \times 10^{7} \mathrm{~V} / \mathrm{m}, E=2 E_{0}$, where $E_{0}=6.9063 \times 10^{7} \mathrm{~V} / \mathrm{m}$. 

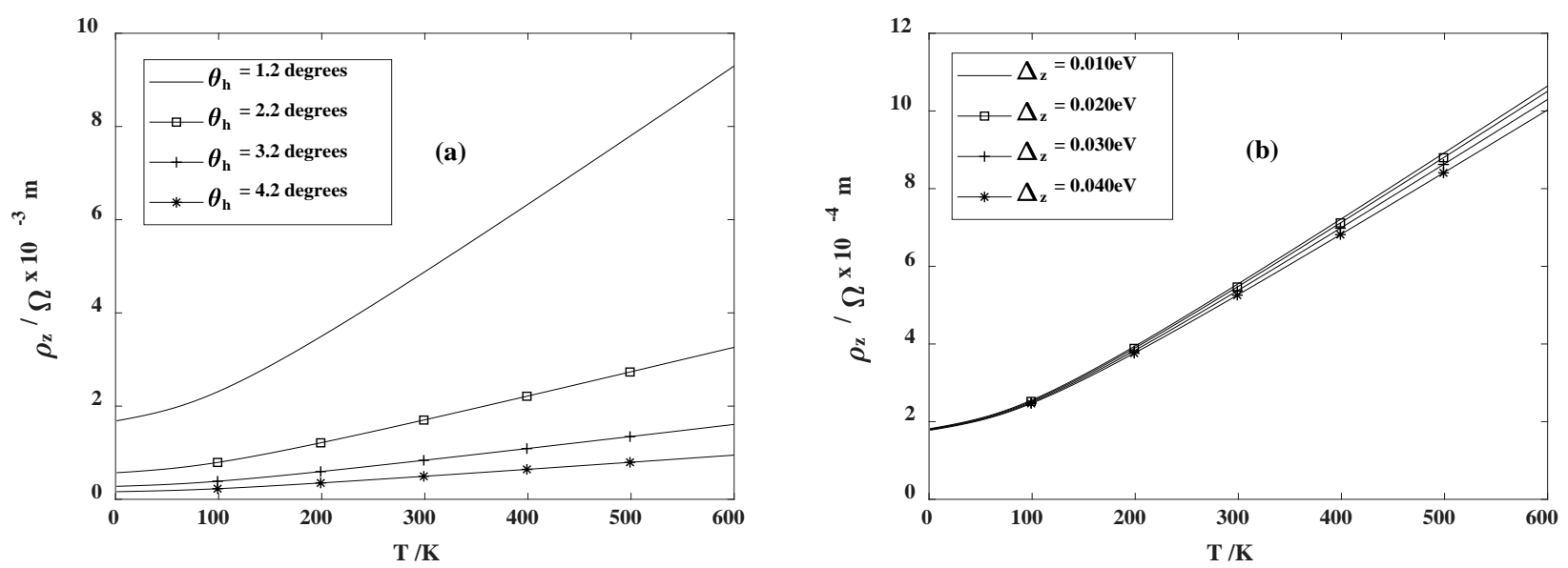

Figure 2. Variation of $\rho_{z}$ with temperature for (a) fixed values of chiral angle, $\theta_{h} . \Delta_{z}=0.024 \mathrm{eV}, \Delta_{y}=0.018 \mathrm{eV}, E_{1}=5.0 \times 10^{7}$ $\mathrm{V} / \mathrm{m}, E=2 E_{0}$, where $E_{0}=6.9063 \times 10^{7} \mathrm{~V} / \mathrm{m}$. (b) The dependence of $\rho_{z}$ on temperature for various fixed values of $\Delta_{z} . \Delta_{y}=0.018$ $\mathrm{eV}, E_{1}=5.0 \times 10^{7} \mathrm{~V} / \mathrm{m}$ and $E=2 E_{0}$, where $E_{0}=6.9063 \times 10^{7} \mathrm{~V} / \mathrm{m}$.
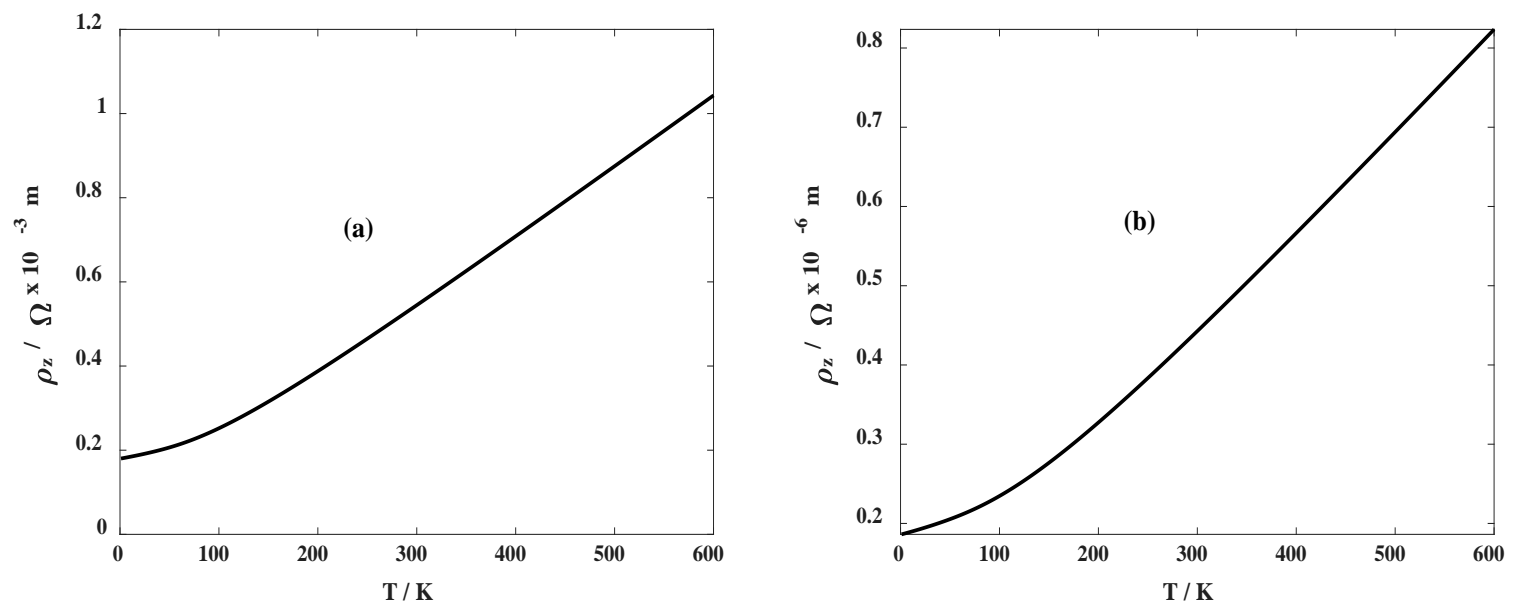

Figure 3. The dependence of $\rho_{z}$ on temperature $T$ for $\Delta_{y}=0.018 \mathrm{eV}, \Delta_{z}=0.024 \mathrm{eV}, E_{1}=5.0 \times 10^{7} \mathrm{~V} / \mathrm{m}, E=2 E_{0}$, where $E_{0}=6.9063 \times 10^{7} \mathrm{~V} / \mathrm{m}$, and chiral angle, $\theta_{h}=4.0^{\circ}$ for the case in which (a) laser is turned and (b) laser is turned off.

On the other hand, when the a.c. source is switched off, $E_{1}=0, a=0, w=0$ and $J_{n}^{2}(a)$ becomes unity and equation (19) reduces to

$$
\sigma_{i}(E)=\frac{e^{2} \tau \Delta_{i} d_{i}^{2} n_{0}}{\hbar^{2}} \frac{I_{1}\left(\Delta_{i}^{*}\right)}{I_{0}\left(\Delta_{i}^{*}\right)} \sum_{n=-\infty}^{\infty} J_{n}^{2}(a)\left[\frac{1}{1+\left(\frac{e d_{i} E_{0}}{\hbar}\right)^{2} \tau^{2}}\right], i=y, z
$$

Figure 3 shows the dependence of axial resistivity of the chiral SWCNTs on temperature in the presence and absence of a laser. Clearly, when the laser source is switched off, $\rho_{z}$ decreases by three orders of magnitude. This indicates that within the temperature range under consideration, the laser field modulates the d. c. field and enhances the momentum and kinetic energy of those electrons which are deficient in energy. 


\section{Conclusion}

Using the semi-classical approach, the axial resistivity of a chiral SWCNT induced by a laser field has been investigated and compared with an earlier work that examined the circumferential resistivity of these unique materials. Our results indicate that the conductivity of SWCNTs is similar for both directions and is significantly influenced by material parameters $\left(\Delta_{y} \Delta_{z}, \theta_{h}\right)$, the strengths of the constant field $E_{0}$ and laser source strength $E_{1}$. The axial resistivity can be increased by increasing the d.c. field strength and decreasing the chiral angle. As observed for the circumferential case, the axial resistivity also steadily and linearly increases with the chiral angle $\theta_{h}$ and real overlapping integral along the axial direction $\Delta_{y}$ at room temperatures. Therefore, SWCNTs can be used in the design of efficient optoelectronic devices.

\section{Conflicts of Interest}

The authors declare no conflicts of interest regarding the publication of this paper.

\section{References}

[1] Iijima, S., Ajayan, P.M. and Ichihashi, T. (1992) Growth Model for Carbon Nanotubes. Physical Review Letters, 69, 3100. https://doi.org/10.1103/PhysRevLett.69.3100

[2] Zhou, W.Y., Bai, X.D., Wang, E.E. and Xie, S.S. (2009) Synthesis, Structure, and Properties of Single-Walled Carbon Nanotubes. Advanced Materials, 21, 4565-4583. https://doi.org/10.1002/adma.200901071

[3] Dekker, C. (1999) Carbon Nanotubes as Molecular Quantum Wires. Physics Today, 52, 22-30. https://doi.org/10.1063/1.882658

[4] Ionel, S., Lepadatu, A.-M., Teodorescu, V.S., Ciurea, M.L., Iancu, V., Dragoman, M., Konstantinidis, G. and Buiculescu, R. (2011) Electrical Behavior of Multi-Walled Carbon Nanotube Network Embedded in Amorphous Silicon Nitride. Nanoscale Research Letters, 6, Article Number: 88. https://doi.org/10.1186/1556-276X-6-88

[5] Purewal, M.S., Hong, B.H., Ravi, A., Chandra, B., Hone, J. and Kim, P. (2007) Scaling of Resistance and Electron Mean Free Path of Single-Walled Carbon Nanotubes. Physical Review Letters, 98, 186808. https://doi.org/10.1103/PhysRevLett.98.186808

[6] Kane, C.L., Mele, E.J., Lee, R.S., Fischer, J.E., Petit, P., Dai, H., Thess, A., et al. (1998) Temperature-Dependent Resistivity of Single-Wall Carbon Nanotubes. EPL (Europhysics Letters), 41, 683. https://doi.org/10.1209/epl/i1998-00214-6

[7] Twum, A., Mensah, S.Y., Edziah, R. and Arthur, A. (2020) Laser Induced Resistivity of Chiral Single Wall Carbon Nanotubes. Journal of Physics Communications, 4, 075011. https://doi.org/10.1088/2399-6528/aba1d4

[8] Mensah, S.Y. and Kangah, G.K. (1992) The Thermoelectric Effect in a Semicon Ductor Superlattice in a Non-Quantized Electric Field. Journal of Physics: Condensed Matter, 4, 919. https://doi.org/10.1088/0953-8984/4/3/031

[9] Slepyan, G.Y., Maksimenko, S.A., Lakhtakia, A., Shenko, O.M.Y. and Gusakov, A.V. (1998) Electronic and Electromagnetic Properties of Nanotubes. Physical Review B, 57, 9485. https://doi.org/10.1103/PhysRevB.57.9485 
[10] Yevtushenko, O.M., Slepyan, G.Y., Maksimenko, S.A., Lakhtakia, A. and Romanov, D.A. (1997) Nonlinear Electron Transport Effects in a Chiral Carbon Nanotube. Physical Review Letters, 79, 1102. https://doi.org/10.1103/PhysRevLett.79.1102

[11] Mensah, S.Y., Allotey, F.K.A., Mensah, N.G. and Nkrumah, G. (2001) Differential Thermopower of a CNT Chiral Carbon Nanotube. Journal of Physics: Condensed Matter, 13, 5653. https://doi.org/10.1088/0953-8984/13/24/310

[12] Vavro, J., Kikkawa, J.M. and Fischer, J.E. (2005) Metal-Insulator Transition in Doped Single-Wall Carbon Nanotubes. Physical Review B, 71, 155410. https://doi.org/10.1103/PhysRevB.71.155410

[13] Yanagi, K., Udoguchi, H., Sagitani, S., Oshima, Y., Takenobu, T., Kataura, H., Ishida, T., Matsuda, K. and Maniwa, Y. (2010) Transport Mechanisms in Metallic and Semiconducting Single-Wall Carbon Nanotube Networks. Acs Nano, 4, 4027-4032. https://doi.org/10.1021/nn101177n 